\title{
Multilocus genotypes of relevance for drug metabolizing enzymes and therapy with thiopurines in patients with acute lymphoblastic leukemia
}

\author{
Gabriele Stocco $^{1,2}{ }^{*}$, Raffaella Franca ${ }^{3}$, Federico Verzegnassi ${ }^{3}$, Margherita Londero $^{4,5}$, Marco Rabusin $^{3}$ and \\ Giuliana Decorti ${ }^{2}$
}

1 Department of Pharmaceutical Sciences, St. Jude Children's Research Hospital, Memphis, TN, USA

${ }^{2}$ Department of Life Sciences, University of Trieste, Trieste, Italy

${ }^{3}$ Institute for Maternal and Child Health IRCCS Burlo Garofolo, Trieste, Italy

${ }^{4}$ Scuola di Dottorato di Ricerca in Scienze della Riproduzione, University of Trieste, Trieste, Italy

${ }^{5}$ Ospedale di San Daniele, Azienda per i Servizi Sanitari 4, Udine, Italy

\section{Edited by:}

Kathrin Klein, Dr. Margarete

Fischer-Bosch-Institute of Clinical

Pharmacology, Germany

Reviewed by:

Branka Zukic, University of Belgrade, Serbia

Mirko Manchia, University of Cagliari, Italy

\section{*Correspondence:}

Gabriele Stocco, Department of Life Sciences, University of Trieste, Via

Fleming 22, Trieste 34127, Italy.

e-mail: gabriele.stocco@stjude.org; stoccog@units.it

Multilocus genotypes have been shown to be of relevance for using pharmacogenomic principles to individualize drug therapy. As it relates to thiopurine therapy, genetic polymorphisms of TPMT are strongly associated with the pharmacokinetics and clinical effects of thiopurines (mercaptopurine and azathioprine), influencing their toxicity and efficacy. We have recently demonstrated that TPMT and ITPA genotypes constitute a multilocus genotype of pharmacogenetic relevance for children with acute lymphoblastic leukemia (ALL) receiving thiopurine therapy. The use of high-throughput genomic analysis allows identification of additional candidate genetic factors associated with pharmacogenetic phenotypes, such as TPMT enzymatic activity: PACSIN2 polymorphisms have been identified by a genome-wide analysis, combining evaluation of polymorphisms and gene expression, as a significant determinant of TPMT activity in the HapMap CEU cell lines and the effects of PACSIN2 on TPMT activity and mercaptopurine induced adverse effects were confirmed in children with ALL. Combination of genetic factors of relevance for thiopurine metabolizing enzyme activity, based on the growing understanding of their association with drug metabolism and efficacy, is particularly promising for patients with pediatric ALL. The knowledge basis and clinical applications for multilocus genotypes of importance for therapy with mercaptopurine in pediatric ALL is discussed in the present review.

Keywords: acute lymphoblastic leukemia, mercaptopurine, pharmacogenetics, multilocus genotypes, TPMT, ITPA, PACSIN2

\section{INTRODUCTION}

The principle of personalized therapy is the identification and application of features associated with treatment response, to select adequate medications and their doses, in order to offer to patients the most effective treatment, with the lower incidence of adverse events (Cheok and Evans, 2006). Among the several features that can be used to personalize therapy, demographic, clinical, and pharmacological ones have been considered. The application of therapy targeted according to these features, related to different treatment outcomes, has greatly improved the effectiveness and safety of therapy, in particular for patients with pediatric cancer, such as acute lymphoblastic leukemia (ALL).

\section{PERSONALIZED THERAPY IMPROVES TREATMENT EFFECTS: THE PARADIGM OF CHILDHOOD ALL}

Optimal use of existing antileukemic agents and improved supportive care in contemporary clinical trials have improved the 5 -year survival rate of childhood ALL above $85 \%$ in developed countries, a disease that was universally fatal in the sixties; moreover, molecular characteristics of leukemia cells have been shown to influence treatment response (Pui and Evans, 2006; Pui et al., 2012).

Pharmacological therapy for childhood ALL consists in protocols in which specific treatment approaches may differ but consistently comprise three major treatment phases: remission induction therapy followed by consolidation/intensification therapy and then continuation/maintenance treatment to eliminate residual leukemic cells (Pui and Evans, 2006). Several medications are used in these treatment phases, comprising various lympholytic and cytotoxic drugs such glucocorticoids (i.e., prednisone, dexamethasone), asparaginase and vincristine, which are particular important for the induction of disease remission. The purine analog mercaptopurine is a key medication for the successful treatment of childhood ALL, in particular for the consolidation and continuation therapies and is used in combination with the folate analog methotrexate: for the success of ALL treatment, the 18-24 months of adequate maintenance therapy comprising mercaptopurine and methotrexate have a key role and are necessary to prolong and consolidate the remission obtained during the initial treatment phases (Pui and Evans, 2006; Paugh et al., 2010; Stocco et al., 2010). 


\section{GENETIC FEATURES MAY INFLUENCE RESPONSE TO THERAPY}

Genetic polymorphisms for genes involved in drug metabolism, transport and molecular mechanism of action can alter the concentration of active metabolites and the molecular function of drugs' targets and therefore the efficacy and safety of pharmacological therapies (Paugh et al., 2011; Pinto et al., 2012). These genetic polymorphisms could therefore function as biomarkers for toxicity and efficacy, allowing the identification of patients with modified sensitivity, because of their genetic characteristics involving drug pharmacokinetics and pharmacodynamics. While many associations between single genetic polymorphisms and drug effects have been clearly demonstrated, showing that inherited genomic variation causes substantial interindividual differences in drug effects, the clinical implementation of these associations is still limited (Relling and Klein, 2011). This is due mainly to the lack of freely available, peer-reviewed, updatable, and detailed gene/drug clinical practice guidelines and even to the very high standards many clinicians and regulators hold for pharmacogenetic evidence (Relling et al., 2010). One of the main efforts to provide these guidelines, which could base the clinical implementation of pharmacogenomics, is that of the Clinical Pharmacogenetics Implementation Consortium (CPIC; Relling and Klein, 2011). CPIC was established in 2009 and consists of members of the Pharmacogenomics Research Network, the main US-based research network in this field, supported by the PharmGKB staff, one of the most important resources for curated pharmacogenomics knowledge (McDonagh et al., 2011), and other affiliated experts in pharmacogenetics, pharmacogenomics and laboratory medicine (Relling and Klein, 2011).

Currently, the CPIC has provided guidelines for pharmacogenetic implementation for 7 medications: abacavir, allopurinol, clopidogrel, codeine, simvastatin, thiopurines, and warfarin (https://www.pharmgkb.org/page/cpic). The process of guidelines definition and preparation is still ongoing and other potential guidelines may be of interest, such as inosine triphosphate pyrophosphatase (ITPA) genetic polymorphism and ribavirin (Fellay et al., 2010), for which a good amount of evidence and replication has been made (Ochi et al., 2010; Thompson et al., 2010; D'Avolio et al., 2012). Moreover, besides CPIC, other research groups have been putting together similar guidelines, such as the European Dutch and German translational pharmacogenomics research teams (Swen et al., 2008, 2011; Schwab and Brauch, 2012).

For the pharmacological therapy of pediatric ALL, several examples have been reported of genetic polymorphisms influencing drug response and toxicity, such as for prednisone polymorphisms of SMARCB1 (Pottier et al., 2007, 2008) and GST-M1 (Marino et al., 2009), for methotrexate solute carrier organic anion transporter family member 1B1 (SLCO1B1) (Trevino et al., 2009; Ramsey et al., 2012), for vincristine ABCB1 and CYP3A4/CYP3A5 (Paugh et al., 2010); however, the only drug - gene pair with a validated guideline published by CPIC that is fully relevant for therapy of pediatric ALL is that of mercaptopurine and thiopurine-Smethyltransferase (TPMT). Indeed, for mercaptopurine, genetic polymorphisms of TPMT have been demonstrated to influence drug metabolism and its effects, constituting one of the most studied and significant example of associations between drug clinical effects and a genetic polymorphism (Paugh et al., 2011).
In lymphoid tissues, mercaptopurine is converted to its active metabolites, the thioguanine nucleotides (TGNs) and is inactivated primarily to methylmercaptopurine by TPMT (Stocco et al., 2010; Zaza et al., 2010). TPMT is encoded by a gene that has non-synonymous single-nucleotide polymorphisms, leading to reduced TPMT activity. In the majority of world populations studied to date, $\sim 1$ in 180 to 1 in 3,700 individuals (depending on ethnicity) inherit two non-functional variants of the TPMT gene, $3-14 \%$ are heterozygous, and the rest are homozygous wild-type. With chronic conventional doses of mercaptopurine, patients who inherit two inactive TPMT alleles universally experience severe myelosuppression, because of accumulation of high levels of cellular TGNs; a high proportion (30-60\%) of patients heterozygous for a TPMT variant allele does not tolerate full doses of mercaptopurine, again because of excessive TGNs. Three TPMT singlenucleotide polymorphisms account for more than $90 \%$ of inactivating alleles and therefore genotyping tests have a high likelihood of being informative. Characterization of TPMT deficiency by genotyping for the most common inactivating single-nucleotide polymorphisms can prospectively identify patients at higher risk of mercaptopurine hematopoietic toxicity; such genotyping is recommended in US Food and Drug Administration-approved labeling (Paugh et al., 2010, 2011).

The diagnosis of TPMT deficiency allows the rational reduction of mercaptopurine dosages while other concurrent cytotoxic agents remain at their usual unadjusted doses, thereby avoiding toxicity without compromising efficacy. For patients with ALL taking mercaptopurine, the CPIC guidelines indicate that these subjects with the homozygous variant should start with drastically reduced dose (i.e., reduce daily dose 10 -fold and reduce frequency to thrice weekly instead of daily) and in case of myelosuppression, emphasis should be on reducing mercaptopurine over other agents; patients heterozygous for TPMT variant alleles (intermediate activity) should start at $30-70 \%$ of full dose and again, in case of myelosuppression, emphasis should be on reducing mercaptopurine over other agents. Patients with normal TPMT should begin therapy with normal starting dose and adjust doses of mercaptopurine (and of any other myelosuppressive therapy) without any special emphasis on mercaptopurine compared to other agents. For all genotypes, in case of dose adjustment, the guidelines specify to allow 2-6 weeks to reach steady state after each dose adjustment, with longer time needed for patients with inactive allele(s) as compared to patients with functional ones (Relling et al., 2011; Table 1). Indeed, it has been shown that, in an ALL protocol using mercaptopurine, prospective adjustment of mercaptopurine based on TPMT status allowed successful treatment of patients with variant TPMT at a reduced dose, with toxicity and efficacy comparable to those in patients with wild-type TPMT (Relling et al., 2006; Stocco et al., 2009).

\section{MULTILOCUS GENOTYPES}

Most of the associations evaluated in the literature and those ready for clinical implementation, i.e. with published curated guidelines, are considering single gene influencing response to a particular drug. However, one of the most important, in terms of relevance and diffusion of drug treatment and potential improvement of guideline application to influence drug use in the clinical setting 


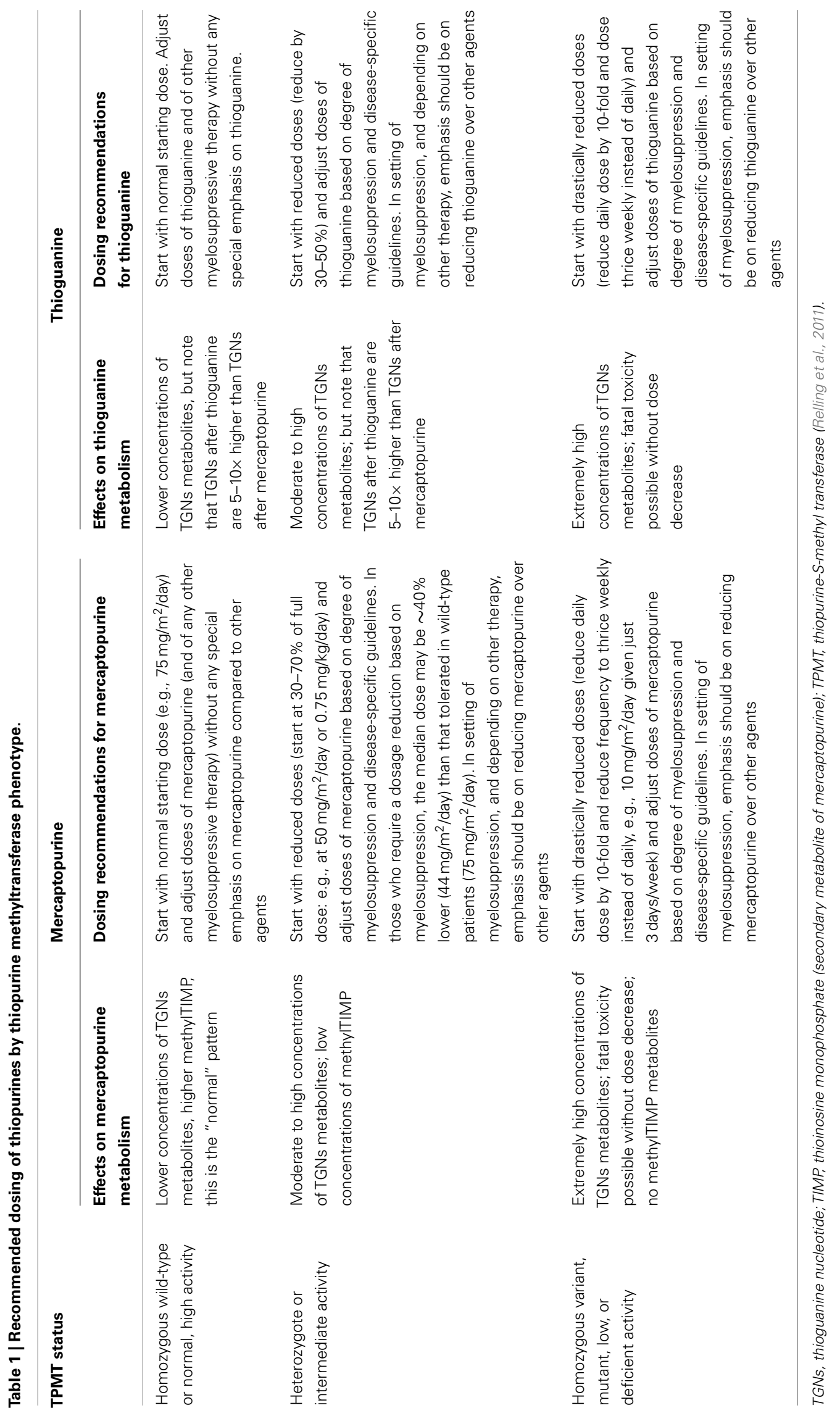


are the guidelines for warfarin, the most commonly used oral anticoagulant worldwide (Johnson et al., 2011); indeed, these guidelines consider genetic variability at two loci: one for the hepatic drug metabolizing enzyme CYP2C9 and one for the target enzyme of warfarin, that is vitamin K-epoxide reductase (VKORC1). CYP2C9 and VKORC1 genetic polymorphisms account for up to 18 and $30 \%$, respectively, of the variance in stable warfarin dose among patients of European ancestry: these common polymorphisms in both genes affect warfarin pharmacokinetics (CYP2C9) and pharmacodynamics (VKORC1) and modulate the therapeutics dose necessary to maintain the optimal level of drug effect (i.e., anticoagulation), preventing the risk of adverse events due to low efficacy or excessive anticoagulation (i.e., respectively thrombosis or bleeding). Combination of CYP2C9 and VKORC1 genetic polymorphisms is important to select the most appropriate dose to start therapy with warfarin: patients are classified by the multilocus genotype in a $2 \times 2$ table, according to the combined effects of the most relevant polymorphisms in each gene (two SNPs for CYP2C9 and one SNP for VKORC1) in three levels of warfarin starting dose; this table is currently inserted in the US Food and Drug's Administration approved warfarin label. More complex algorithms, comprising even relevant demographic and clinical patient's characteristics affecting warfarin efficacy, such as age, smoking status and interacting drugs have been developed; some of these algorithms consider even additional genetic information besides CYP2C9/VKORC1 multilocus genotype, such as polymorphisms of the CYP4F2 and GGCX genes. It has been shown that warfarin dosing criteria considering genetics outperform non-genetic clinical algorithms and are particularly beneficial for patients requiring relatively low or high doses of the medication (i.e., $<21 \mathrm{mg} /$ week or $>49 \mathrm{mg} /$ week), that however are $\sim 40 \%$ of all patients: thanks to genetic based dose selection, these patients reach their optimal dose level more quickly and therefore with a lower risk of developing adverse events. Moreover, these criteria are particularly important for patients starting warfarin therapy, while are less useful for already established treatments. The development of these important therapeutic guidelines, considering a multilocus genotype affecting warfarin dose requirements illustrates how genetic information in more than one gene can be of clinical relevance to guide therapy for a single medication. Other similar guidelines considering multiple loci are in development, such as CYP2D6/CYP2C9 multilocus genotype for tricylic antidepressants (Consortium, 2012).

\section{MULTILOCUS GENOTYPES OF RELEVANCE FOR THERAPY PERSONALIZATION OF PEDIATRIC ALL TPMT AND ITPA AND MAINTENANCE THERAPY FOR PEDIATRIC ALL}

In addition to TPMT, other genetic factors may alter the effects of mercaptopurine, although their clinical importance has not been as well characterized. It has been shown that once mercaptopurine treatment for childhood ALL is individualized for $T P M T$, the effect of genetic polymorphisms in inosine triphosphate pyrophosphatase (ITPA) emerges (Stocco et al., 2010). ITPA is an enzyme that catalyzes the hydrolysis of inosine triphosphate (ITP) to inosine monophosphate (IMP). IMP is a central intermediate in purine metabolism and can be converted to ITP and to ATP via AMP or to GTP via GMP. The putative role of ITPA is to protect cells from the accumulation of potentially harmful nucleotides, such as ITP or deoxy-ITP, which may be incorporated into nucleic acids; indeed, it has been demonstrated by knock-down experiments performed in HeLa cells, that ITPA has a significant role in preventing base analog induced apoptosis, DNA damage, and mutagenesis in human cells (Menezes et al., 2012). In humans, ITPA displays a genetically determined polymorphic activity (Marsh and Van Booven, 2009; Stocco et al., 2010). Characterization of ITPA haplotype structure has shown that the SNP rs1127354 is the most relevant polymorphism in determining ITPA low enzymatic activity (von Ahsen et al., 2008; Stocco et al., 2010). Our recent study assessed the influence of nonfunctional variant alleles of TPMT and ITPA on mercaptopurine metabolism and toxicity in patients with ALL whose mercaptopurine doses were adjusted based on TPMT genotype (Stocco et al., 2009). This study revealed that the cumulative incidence of severe adverse effects (grade 3-4 febrile neutropenia) in patients receiving maintenance therapy that includes mercaptopurine individualized for TPMT is significantly greater among patients who have inherited an ITPA variant allele; this association remained significant when the analysis was limited to only life threatening events (i.e., grade 4 fever and neutropenia). Our recent study has documented that inheritance of a non-functional variant allele for either TPMT or ITPA is associated with significant modification in the metabolism of mercaptopurine during treatment of ALL. Although the importance of the TPMT genetic polymorphism is very well known and characterized, this was the first report showing a significant effect of the ITPA genetic polymorphism in the context of mercaptopurine therapy that has been individualized based on TPMT genotype. We documented significantly higher concentrations of the methylated nucleotide metabolites of mercaptopurine in leukemia cells and erythrocytes of patients who have inherited a non-functional ITPA allele. In contrast, the inheritance of a variant ITPA allele was not associated with differences in TGN concentrations in either leukemia cells or erythrocytes. Although ITPA is known to be involved in mercaptopurine metabolism, the mechanism by which ITPA variant alleles influence the accumulation of methylated thionucleotides has not been fully elucidated (Stocco et al., 2009, 2010).

A recent study has replicated the observation of the effects of the combined TPMT and ITPA genotype on the mercaptopurine pharmacokinetics and in particular on the concentration of methylated-mercaptopurine-nucleotides: among 66 children with ALL, treated according to EORTC 58951 protocol, comprising mercaptopurine at a dose of $50 \mathrm{mg} / \mathrm{m}^{2} /$ day and methotrexate at a dose of $20 \mathrm{mg} / \mathrm{m}^{2} /$ week, methylated-mercaptopurine-nucleotides concentrations were low in patients with TPMT variant/ITPA wild-type multilocus genotype, intermediate in wild-type/wildtype patients and high in patients with wild-type TPMT/ITPA variant (Adam de Beaumais et al., 2010).

It is known that ethnic differences for genotype frequencies may influence treatment efficacy in ALL: for example, it has been reported that the component of genomic variation that cosegregated with Native-American ancestry was associated with risk of relapse, even after adjusting for known prognostic factors (Yang et al., 2011). The allele frequencies of TPMT and ITPA polymorphisms show significant inter-ethnic variability: in 
particular for rs1127354 of ITPA allele frequency of the variant is known to be $\sim 20 \%$ in Asian populations, $\sim 6 \%$ in Caucasians, and $\sim 2 \%$ in Hispanics, while for TPMT, the most common variants (rs1142345, rs1800460 and rs1800462) have a frequency of $~ 1 \%$ in Asians, $\sim 5 \%$ in Caucasians, and $\sim 10 \%$ in Hispanics. Therefore, it is interesting that for TPMT and ITPA, frequencies of the variant alleles associated with different metabolism of mercaptopurine, seem to be almost reversal in the two populations (Marsh and Van Booven, 2009) and ITPA variants seem to be predominant in the Asian population. Indeed several recent studies of patients of Asian ethnicity seem to underline significant effects of ITPA polymorphisms on thiopurines' efficacy and toxicity in patients with ALL, but even when these medications are used as immunosuppressants in other pathologies (Okada et al., 2009; Yamamoto et al., 2010). For children with ALL, a recent study in 90 Indian patients, on maintenance therapy according to the MCP-841 protocol (Advani et al., 1999) with mercaptopurine at a dose of $75 \mathrm{mg} / \mathrm{m}^{2}$ for 12 weeks, showed an independent role for both TPMT and ITPA in terms of association with the incidence of hematological toxicity; moreover, the multilocus genotype TPMT/ITPA was associated with a gene-dosage effect: percentage of reduction in total leukocyte count (i.e., the average leukocyte count on days 43, 71, and 99 of maintenance therapy) resulted in $\sim 40 \%$ for a patient with a wild-type genotype at both the TPMT and ITPA loci and increased proportionally to the number of risk alleles (i.e., variant inactive alleles for TPMT or ITPA) up to almost $70 \%$ in patients with three or more risk alleles at the TPMT and ITPA loci (Dorababu et al., 2012a). Analysis of epistasis by multifactor dimensionality reduction (Hahn et al., 2003) confirmed synergistic interactions between TPMT and ITPA variant alleles, in terms of their association with hematological toxicity during ALL maintenance therapy for this cohort of Indian children. Another recent study considered 100 Korean patients with pediatric ALL and evaluated in these patients 18 loci in 16 candidate genes of pharmacogenetic interest, including TPMT and ITPA, and their association with survival rate. Even if this study did not seem to confirm a strong difference for TPMT and ITPA gene variants between a western population of reference and the Korean patients, there was a significant effect of ITPA genotype, but not of TPMT, on the event free survival rate, which was lower in ITPA variants. TPMT genotype was however associated with the tolerance of mercaptopurine and methotrexate, evaluated as the dose of the medications used during the last cycle of maintenance therapy: indeed, as expected, patients with variant TPMT were selected to be treated with lower doses of mercaptopurine; unfortunately, data about the effect of ITPA genotype on the doses of antimetabolites was not reported (Kim et al., 2012).

Tanaka et al. (2012) have measured the activity of ITPA in 65 Japanese children with pediatric ALL, showing that patients with lower activity of this enzyme tolerated lower doses of mercaptopurine during maintenance therapy and presented increased probability of hepatoxicity.

In Asian populations, therefore, polymorphisms of ITPA seem to be of particular relevance for the effects of mercaptopurine in children with ALL, given the low incidence of patients with variant TPMT, compared to patients of Caucasian ethnicity (Marsh and Van Booven, 2009). However, it is known that other genetic polymorphisms may be of particular importance for Asian patients, such as SNP rs3765534 in the transporter MRP4, that is polymorphic only in patients of Asian ethnicity and that has been shown to modulate thiopurines intracellular levels by regulating the efflux of the thionucleotides (Krishnamurthy et al., 2008; Stocco et al., 2010).

On these bases, to understand the pharmacogenetics and improve treatment with thiopurines in the Asian populations, larger prospective studies are needed, considering even multilocus genotypes at loci of known relevance, such as TPMT, ITPA, and MRP4.

\section{MULTILOCUS GENOTYPE TPMT - SLCO1B1 - PACSIN2 AND EFFECTS ON SEVERE MUCOSITIS DURING CONSOLIDATION THERAPY FOR PEDIATRIC ALL}

During consolidation therapy for pediatric ALL, patients are treated with weekly $24 \mathrm{~h}$ infusions of high dose methotrexate, up to $5 \mathrm{~g} / \mathrm{m}^{2}$ and daily oral mercaptopurine with doses that range from 25 to $50 \mathrm{mg} / \mathrm{m}^{2}$. Therapy with this association of antimetabolites has a very important role in preventing the relapse of the disease, after remission induction; however consolidation therapy is associated with the development of adverse effects, in particular gastrointestinal toxicity, such as stomatitis and mucositis, which cause major discomfort for the patient and can be severe, preventing the children from normal food intake and requiring parenteral nutrition. To avoid adverse events related to consolidation therapy, one of the most common approaches used in therapeutic protocols for ALL worldwide is the administration of leucovorin, a source of folic acid, that contrasts the cytotoxic effects of methotrexate and its association with mercaptopurine. Most protocols for ALL worldwide measure the concentration of methotrexate in patients' blood at the end of each infusion and administer leucovorin if methotrexate is then still present at significant concentrations: for example, in the Italian AIEOP-BFM ALL 2000 protocol, leucovorin was administered every $6 \mathrm{~h}$ at a dose of $7.5 \mathrm{mg} / \mathrm{m}^{2}$, if methotrexate concentration resulted higher than $0.5 \mu \mathrm{mol} / \mathrm{l}$ at $48 \mathrm{~h}$ from the beginning of the infusion and until methotrexate concentration dropped below $0.25 \mu \mathrm{mol} / 1$ (Conter et al., 2010; Schrappe et al., 2011). Consolidation therapy lasts from 2 weeks up to 3 months depending on the treatment protocol and therefore the length of the therapy is too short to implement therapeutic monitoring of mercaptopurine metabolites concentration, which are useful when the drug is taken for at least 2 months (Lennard and Lilleyman, 1989). Advanced protocols for treatment of ALL, developed at St. Jude Children's Research Hospital in Memphis, evaluate the clearance of methotrexate during the infusion and either adjust the speed of infusion of the drug to a target concentration in the subsequent course (Total XV protocol) or in the same course (Total XVI protocol). This procedure requires a quick and efficient turnaround of the samples for the measurement of methotrexate concentration, which need to be analyzed in a few hours timeframe, so that the clearance of methotrexate can be estimated during the infusion, and the medication's administration speed can be adapted to reach the desired concentration threshold (i.e., $33 \mu \mathrm{M}$ for low risk patients, $65 \mu \mathrm{M}$ for standard-high risk patients; Pui et al., 2009). Therapeutic monitoring of methotrexate during consolidation therapy has significantly improved patients' 
tolerance to this association treatment with antimetabolites; however, about $5 \%$ of pediatric patients still develop severe stomatitis/mucositis, with consequences that can be life threatening. The study of pharmacogenetic determinants of severe gastrointestinal (GI) toxicity during consolidation therapy has lead to major breakthroughs in recent years, which hopefully will lead to even better treatment of patients with ALL, completely preventing the occurrence of this major adverse event. In particular, a recent genome-wide study analyzed 500,568 germline singlenucleotide polymorphisms to identify how inheritance affects methotrexate plasma disposition among 434 children with ALL who received 3,014 courses of methotrexate at $2-5 \mathrm{~g} / \mathrm{m}^{2}$ (Trevino et al., 2009). This study lead to the identification of polymorphisms in SLCO1B1, as the most significant associations ( $p$-value $\left.<10^{-9}\right)$ with methotrexate clearance, even after adjusting for age, race, sex, and methotrexate regimen. In particular, the most significant polymorphism was the intronic rs11045879, which is in linkage disequilibrium with the functional SNP rs4149056; these same polymorphisms were associated even with severe GI toxicity during consolidation therapy, mostly severe stomatitis and mucositis. This observation was confirmed by subsequent studies (LopezLopez et al., 2011; Ramsey et al., 2012). Therefore SLCO1B1 polymorphisms are significant determinants for the occurrence of severe GI toxicity and in particular stomatitis/mucositis, during consolidation therapy for pediatric ALL, by an effect on methotrexate disposition: indeed the gene product of SLCO1B1 is a transporter which mediates the sodium-independent uptake of organic anions such as methotrexate and may play an important role in the clearance of bile acids and organic anions.

Another relevant study has considered the role of genetic determinants of mercaptopurine toxicity during consolidation therapy, together with SLCO1B1: this study on adverse effects considered 189 children with ALL and evaluated the association between genetic determinants of TPMT activity in patients and the incidence of severe GI toxicity. The frequency of GI toxicity (grade 3-4 mucositis) in this population was $8.5 \%$; among these patients, deficiency in TPMT activity predisposed to an increased incidence of severe GI toxicity during consolidation therapy which included methotrexate $\left(2 \mathrm{~g} / \mathrm{m}^{2} /\right.$ week $)$ and mercaptopurine $\left(75 \mathrm{mg} / \mathrm{m}^{2} /\right.$ day, in all patients during consolidation therapy, regardless of TPMT genotype). Indeed, among nine patients with a variant TPMT allele, the frequency of GI toxicity was 33\%, compared with $7.2 \%$ in patients with wild-type TPMT. As previously reported (Trevino et al., 2009), the SLCO1B1 SNP rs11045879 was also associated with the incidence of GI toxicity: indeed none of the patients with the SLCO1B1 CC or CT genotype had GI toxicity, whereas $11.8 \%$ of the patients with the wild-type SLCO1B1 TT genotype had this side effect. Moreover, this study identified, through the HapMap model system, an additional determinant of TPMT activity, the PACSIN2 gene which resulted as the highest correlated gene to TPMT activity, in an analysis combining polymorphisms and expression, all measured in the 30 HapMap CEU trios (Stocco et al., 2012). The most significant PACSIN2 SNP in the HapMap analysis for TPMT activity, rs2413739, was also significantly associated with TPMT activity in patients with ALL, independently from TPMT genotype: the CC genotype for the rs2413739 SNP displayed a higher TPMT activity in comparison to the TT genotype. Moreover, PACSIN2 SNP rs2413739 also had a significant association with GI toxicity during consolidation therapy: the frequency of toxicity was $2.1,9.1$, and $13.2 \%$, respectively, for the CC, CT, and TT genotype (Stocco et al., 2012). The effects of PACSIN2 polymorphism on the incidence of severe mucositis during consolidation therapy for pediatric ALL were confirmed in another cohort of patients, considering 67 cases developing the adverse event during therapy according to the protocol AIEOP-BFM ALL 2000, which involves four weekly infusion of methotrexate at the dose of $2 \mathrm{~g} / \mathrm{m}^{2}$ and concomitant daily treatment with mercaptopurine at the dose of $25 \mathrm{mg} / \mathrm{kg}$. Analysis in the validation cohort was done by a case-control design and each case was matched to two controls from the same protocol based on sex, age, ALL lineage and ALL risk classification, confirming a significant effect of PACSIN2 SNP rs2413739 on the incidence of severe mucositis during the consolidation therapy of pediatric ALL (Stocco et al., 2012). Interestingly, in the discovery cohort, the effects of TPMT, SLCO1B1 and PACSIN2 polymorphisms were independent from each other, both in a multivariate logistic regression model and in a classification and regression tree analysis and could be combined in a multilocus genotype of potential importance to predict the incidence of severe mucositis in children with ALL treated with consolidation therapy comprising the combination of methotrexate and mercaptopurine (Figure 1).

\section{GENOME-WIDE ANALYSIS OF SNPS ASSOCIATED TO CLINICAL RESPONSE IN PEDIATRIC ALL: IMPLICATIONS FOR THE PHARMACOGENETICS OF MERCAPTOPURINE}

Genome-wide analysis, if adequately powered, has great potential in elucidating and understanding the genomic component associated with interindividual differences in phenotypes, even of pharmacogenetic interest. This has been shown to be true in model systems like the HapMap cell lines, in which statistical power is obtained mainly by combining genomic information at the level of gene expression and genetic polymorphisms, with the advantage that the phenotypes can be characterized with great accuracy and consistency (Wheeler and Dolan, 2012). This has led for example to the identification of PACSIN2 as a significant determinant of TPMT activity in the cell lines, with effects reproducible in patients with ALL mentioned above (Stocco et al., 2012). However, the greatest potential of the genome-wide approach resides really in the analysis of patients' samples: if the study is adequately designed and powered and the phenotypes are well collected, this approach can provide unpredictable insights on the phenotype of interest, potentially leading to major breakthroughs in the understanding of the genomic basis of inter-patient variability, even of pharmacogenetic traits. Several such examples exist in the literature: besides the already mentioned role of SLCO1B1 in the disposition of methotrexate in children with ALL (Trevino et al., 2009), this same transporter was shown to be involved in statins' induced myopathy (Link et al., 2008); another example of genome-wide studies is the discovery of a role for ITPA in anemia induced by the anti-viral agent ribavirin (Fellay et al., 2010). 


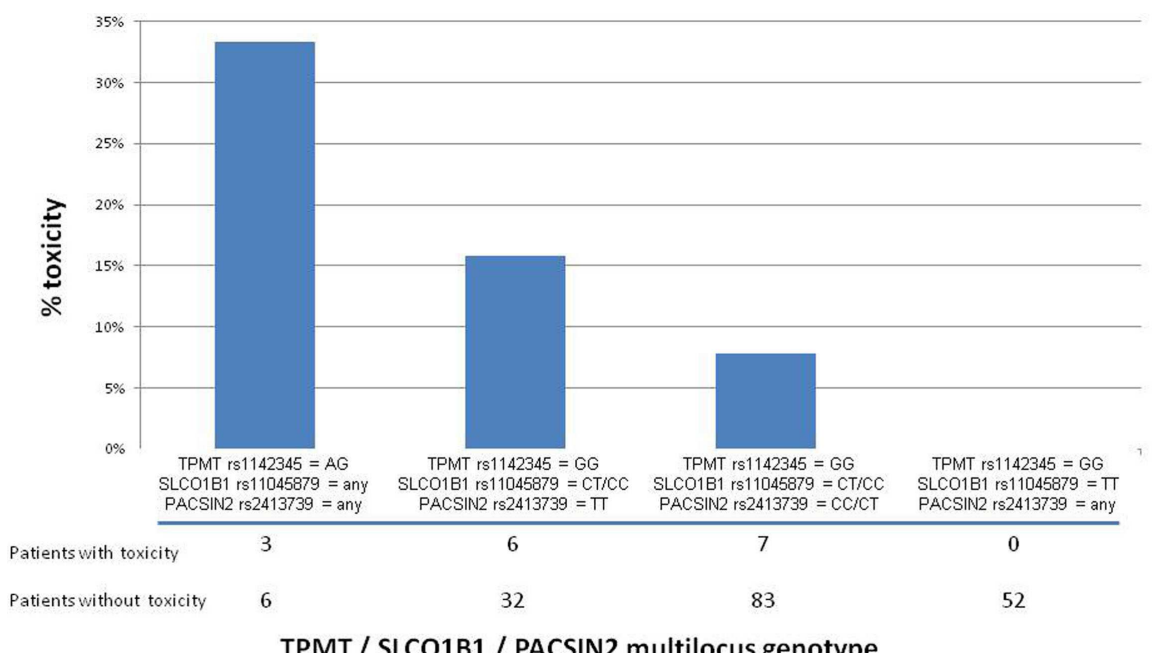

TPMT / SLCO1B1 / PACSIN2 multilocus genotype

p-value, Chi-squared test $=0.0021$

FIGURE 1 | Barplot reporting the percentage of patients developing severe (Grade 3-4) GI toxicity during consolidation therapy in patients with ALL treated according to the St Jude Total 13B protocol as a function of TPMT rs1142345 / SLC01B1 rs11045879 / PACSIN2 rs2413739 multilocus genotype (Stocco et al., 2012).

While in pediatric ALL genome-wide interrogation is complicated by the relative small number of patients available, St. Jude Children's Hospital was able to publish some genome-wide studies on leukemia pharmacogenetics, thanks to access to uniformly treated and well characterized patients and phenotypes (Trevino et al., 2009; Yang et al., 2009, 2012; Kawedia et al., 2011). Among these genome-wide studies of pharmacogenetic interest, some have generated data that could be of particular interest for the identification of multilocus genotypes of relevance for the treatment of ALL with thiopurines. In particular, these studies considered genetic polymorphisms associated with outcome to therapy evaluated as minimal residual disease (MRD) (Yang et al., 2009) or disease relapse (Yang et al., 2012); while these very important clinical phenotypes of patients with ALL are not related directly only to mercaptopurine, the genetic features identified are related even to disposition of antileukemic drugs and may be of relevance for mercaptopurine effects too and should be therefore considered.

The study on MRD considered two independent cohorts of children with newly diagnosed ALL: 318 patients in St Jude Total Therapy protocols XIIIB and XV and 169 patients in Children's Oncology Group trial P9906. This study identified 102 SNPs associated with MRD in both cohorts, including five SNP in interleukin 15 (IL15). Twenty one of these SNPs were also associated with drug disposition (evaluated as methotrexate clearance, etoposide clearance, or methotrexate polyglutamates concentration), generally linking greater drug exposure with MRD eradication. While concentration of mercaptopurine metabolites was not evaluated in this study, the effects on the disposition of methotrexate, that is associated with mercaptopurine both during consolidation and maintenance therapy, suggest that these SNPs may be of interest to build multilocus genotypes useful for therapy personalization of pediatric ALL also with mercaptopurine.

\section{FURTHER DEVELOPMENT OF MULTILOCUS GENOTYPES: EPISTASIS AND GENE-ENVIRONMENT INTERACTIONS}

Phenotypes of pharmacogenetic interest are complex, particularly those describing patients' response to a medication, both in terms of efficacy and incidence of adverse events: it is likely that different genetic features, together with environmental factors, contribute to the interindividual variability of these phenotypes. Indeed, it is known that the effect of genetic polymorphisms is stronger when it refers to a pharmacokinetic phenotype and strength of the association reduces with the increasing complexity of the phenotype: for example, the effect of TPMT genotype is extremely strong on TPMT activity and the strength of the association is reduced, while still significant, considering more complex phenotypes such as the concentration of mercaptopurine metabolites and, even more, considering parameters of clinical response to the medication: the thinning of the association strength is due to the increasing complexity of the phenotype and the augmented potential role of environmental and additional genetic factors (Relling et al., 2011). Moreover, for complex phenotypes such as the response to a medication, the effects of a genetic factor may depend on other genetic variations and environmental factors, a phenomenon that is defined respectively as gene-gene interaction/epistastis or as gene-environment interaction (Moore and Williams, 2009). Methods have been developed to study consistently and efficiently the role of multiple genetic and environmental factors on complex phenotypes defined by discrete traits, such as those of pharmacogenetic interest (e.g., clinical response to a medication or occurrence of adverse events; Hahn et al., 2003; Gilbert-Diamond and Moore, 2011). This method is called multifactor dimensionality reduction (MDR) and allows collapsing multi-dimensional genetic information into a single dimension, thus permitting the detection of epistasis: MDR is a non-parametric method and 
interactions are detected by a constructive induction approach, in particular by classifying multiple loci as high risk or low risk, depending on whether they are more common in affected or in unaffected subjects; this pooling allows reducing the dimensionality of the multilocus data to one dimension (Hahn et al., 2003). The new multilocus genotype variable is then evaluated for its ability to classify and predict the phenotype of interest (i.e., drug response): different approaches have been used to perform these computations; originally, however, it was done by cross-validation and permutation testing and recently extensions and variations of the method have been developed, which allow the calculation of odds ratios and application of Fisher's test to increase model robustness (Moore and Williams, 2009). Interestingly, it has been reported that MDR allows the identification of significant genegene interaction in the absence of a statistically significant main effect by a single genotype; moreover, it was mathematically proved that MDR is the best method to discriminate multilocus genotypes for clinical endpoints. MDR has been successfully applied to detecting gene-gene and gene-environment interactions for a wide variety of different complex phenotypes, such as incidence of human diseases and other clinical endpoints (Gilbert-Diamond and Moore, 2011). Recently this method has been applied even for studies of pharmacogenomics for thiopurines and methotrexate (Dervieux et al., 2012; Dorababu et al., 2012a,b; Kim et al., 2012), even if its application to this field is still limited and there is great

\section{REFERENCES}

Adam de Beaumais, T., Dervieux,

T., Fakhoury, M., Medard, Y., Azougagh, S., Zhang, D., et al. (2010). The impact of high-dose methotrexate on intracellular 6mercaptopurine disposition during interval therapy of childhood acute lymphoblastic leukemia. Cancer Chemother. Pharmacol. 66, 653-658.

Advani, S., Pai, S., Venzon, D., Adde, M., Kurkure, P. K., Nair, C. N., et al. (1999). Acute lymphoblastic leukemia in India: an analysis of prognostic factors using a single treatment regimen. Ann. Oncol. 10, 167-176.

Cheok, M. H., and Evans, W. E. (2006). Acute lymphoblastic leukaemia: a model for the pharmacogenomics of cancer therapy. Nat. Rev. Cancer 6, 117-129.

Consortium, C. P. I. (2012). Clinical Pharmacogenetics Implementation Consortium (CPIC) Gene-drug pairs [Online]. Available at: http://www.pharmgkb.org/page/ cpicGeneDrugPairs [accessed Oct 22, 2012].

Conter, V., Bartram, C. R., Valsecchi, M. G., Schrauder, A., PanzerGrumayer, R., Moricke, A., et al. (2010). Molecular response to treatment redefines all prognostic factors in children and adolescents with
B-cell precursor acute lymphoblastic leukemia: results in 3184 patients of the AIEOP-BFM ALL 2000 study. Blood 115, 3206-3214.

D’Avolio, A., Ciancio, A., Siccardi, Simiele, M., et al. (2012). Inosine triphosphatase polymorphisms and ribavirin pharmacokinetics as determinants of ribavirinassociate anemia in patients receiving standard anti-HCV treatment. Ther. Drug. Monit. 34, 165-170.

Dervieux, T., Wessels, J. A., Kremer, J. M., Padyukov, L., Seddighzadeh, M., Saevarsdottir, S., et al. (2012). Patterns of interaction between genetic and nongenetic attributes and methotrexate efficacy in rheumatoid arthritis. Pharmacogenet. Genomics 22, $1-9$.

Dorababu, P., Nagesh, N., Linga, V. G., Gundeti, S., Kutala, V. K., Reddanna, P., et al. (2012a). Epistatic interactions between thiopurine methyltransferase (TPMT) and inosine triphosphate pyrophosphatase (ITPA) variations determine 6mercaptopurine toxicity in Indian children with acute lymphoblastic leukemia. Eur. J. Clin. Pharmacol. 68, 379-387. G., Gundeti, S., Nagesh, N., Kutala, M., Smedile, A., Baietto, L.,

Dorababu, P., Naushad, S. M., Linga, V.

potential for discovery, in particular to detect and elucidate multilocus genotypes associated with genome-wide studies of complex pharmacogenetic phenotypes.

\section{CONCLUSION}

Consideration of genetic biomarkers can improve therapy of pediatric ALL: the role of TPMT genetic polymorphism on mercaptopurine induced toxicity in children with ALL has been clearly defined and clinical guidelines have been developed to tailor treatment with this medication on the basis of TPMT status. Multilocus genotypes have been shown to be able to increase the amount of interindividual variability in a phenotype of clinical relevance explained: for example, the incidence of severe GI toxicity during consolidation therapy has been shown recently to be independently related to TPMT, SLCO1B1, and PACSIN2 genetic polymorphisms. Identification, proper testing, and validation of multilocus genotypes hold great potential in further refining the clinical utility of pharmacogenetics to improve treatment of children with ALL by reducing treatment-related adverse events.

\section{ACKNOWLEDGMENTS}

Dr. Stocco was a post-doctoral research fellow at St. Jude Children's Research Hospital when this manuscript was developed. We thank Dr. William E. Evans, CEO of SJCRH, for his helpful comments during the preparation of this manuscript.

V. K., et al. (2012b). Genetic variants of thiopurine and folate metabolic pathways determine 6-MPmediated hematological toxicity in childhood ALL. Pharmacogenomics 13, 1001-1008.

Fellay, J., Thompson, A. J., Ge, D. Gumbs, C. E., Urban, T. J., Shianna, K. V., et al. (2010). ITPA gene variants protect against anaemia in patients treated for chronic hepatitis C. Nature 464, 405-408.

Gilbert-Diamond, D., and Moore, J. H. (2011). Analysis of gene-gene interactions. Curr. Protoc. Hum. Genet. Chap. 1, Unit1 14.

Hahn, L. W., Ritchie, M. D., and Moore, J. H. (2003). Multifactor dimensionality reduction software for detecting gene-gene and geneenvironment interactions. Bioinformatics 19, 376-382.

Johnson, J. A., Gong, L., Whirl-Carrillo, M., Gage, B. F., Scott, S. A., Stein, C. M., et al. (2011). Clinical pharmacogenetics implementation consortium guidelines for CYP2C9 and VKORC1 genotypes and warfarin dosing. Clin. Pharmacol. Ther. 90, 625-629.

Kawedia, J. D., Kaste, S. C., Pei, D., Panetta, J. C., Cai, X., Cheng, C., et al. (2011). Pharmacokinetic, pharmacodynamic, and pharmacogenetic determinants of osteonecrosis in children with acute lymphoblastic leukemia. Blood 117, 2340-2347, quiz 2556.

Kim, H., Kang, H. J., Kim, H. J., Jang, M. K., Kim, N. H., Oh, Y., et al. (2012). Pharmacogenetic analysis of pediatric patients with acute lymphoblastic leukemia: a possible association between survival rate and ITPA polymorphism. PLoS ONE 7:e45558. doi:10.1371/journal.pone.0045558

Krishnamurthy, P., Schwab, M., Takenaka, K., Nachagari, D., Morgan, J., Leslie, M., et al. (2008). Transportermediated protection against thiopurine-induced hematopoietic toxicity. Cancer Res. 68, 4983-4989.

Lennard, L., and Lilleyman, J. S. (1989). Variable mercaptopurine metabolism and treatment outcome in childhood lymphoblastic leukemia. J. Clin. Oncol. 7, 1816-1823.

Link, E., Parish, S., Armitage, J., Bowman, L., Heath, S., Matsuda, F., et al. (2008). SLCO1B1 variants and statin-induced myopathy - a genome wide study. N. Engl. J. Med. 359, 789-799.

Lopez-Lopez, E., Martin-Guerrero, I., Ballesteros, J., Pinan, M. A., GarciaMiguel, P., Navajas, A., et al. (2011). Polymorphisms of the SLCO1B1 gene predict methotrexate-related toxicity in childhood acute lymphoblastic leukemia. Pediatr. Blood Cancer 57, 612-619. 
Marino, S., Verzegnassi, F., Tamaro, P., Stocco, G., Bartoli, F., Decorti, G., et al. (2009). Response to glucocorticoids and toxicity in childhood acute lymphoblastic leukemia: role of polymorphisms of genes involved in glucocorticoid response. Pediatr. Blood Cancer 53, 984-991.

Marsh, S., and Van Booven, D. J. (2009). The increasing complexity of mercaptopurine pharmacogenomics. Clin. Pharmacol. Ther. 85, 139-141.

McDonagh, E. M., Whirl-Carrillo, M., Garten, Y., Altman, R. B., and Klein, T. E. (2011). From pharmacogenomic knowledge acquisition to clinical applications: the PharmGKB as a clinical pharmacogenomic biomarker resource. Biomark. Med. 5, 795-806.

Menezes, M. R., Waisertreiger, I. S., Lopez-Bertoni, H., Luo, X., and Pavlov, Y. I. (2012). Pivotal role of inosine triphosphate pyrophosphatase in maintaining genome stability and the prevention of apoptosis in human cells. PLoS ONE 7:e32313. doi:10.1371/journal.pone.0032313

Moore, J. H., and Williams, S. M. (2009). Epistasis and its implications for personal genetics. Am. J. Hum. Genet. 85, 309-320.

Ochi, H., Maekawa, T., Abe, H., Hayashida, Y., Nakano, R., Kubo, M., et al. (2010). ITPA polymorphism affects ribavirin-induced anemia and outcomes of therapy a genome-wide study of Japanese $\mathrm{HCV}$ virus patients. Gastroenterology 139, 1190-1197.

Okada, Y., Nakamura, K., Hiromura, K., Nojima, Y., Horiuchi, R., and Yamamoto, K. (2009). Pro32Thr polymorphism of inosine triphosphate pyrophosphatase gene predicts efficacy of low-dose azathioprine for patients with systemic lupus erythematosus. Clin. Pharmacol. Ther. 85, 527-530.

Paugh, S. W., Stocco, G., and Evans, W. E. (2010). Pharmacogenomics in pediatric leukemia. Curr. Opin. Pediatr. 22, 703-710.

Paugh, S. W., Stocco, G., McCorkle, J. R., Diouf, B., Crews, K. R., and Evans, W. E. (2011). Cancer pharmacogenomics. Clin. Pharmacol. Ther. 90, 461-466.

Pinto, N., Cohn, S. L., and Dolan, M. E. (2012). Using germline genomics to individualize pediatric cancer treatments. Clin. Cancer Res. 18, 2791-2800.

Pottier, N., Cheok, M. H., Yang, W., Assem, M., Tracey, L., Obenauer, J. C., et al. (2007). Expression of SMARCB1 modulates steroid sensitivity in human lymphoblastoid cells: identification of a promoter SNP that alters PARP1 binding and SMARCB1 expression. Hum. Mol. Genet. 16, 2261-2271.

Pottier, N., Yang, W., Assem, M., Panetta, J. C., Pei, D., Paugh, S. W., et al. (2008). The SWI/SNF chromatinremodeling complex and glucocorticoid resistance in acute lymphoblastic leukemia. J. Natl. Cancer Inst. 100 , 1792-1803.

Pui, C. H., Campana, D., Pei, D., Bowman, W. P., Sandlund, J. T., Kaste, S. C., et al. (2009). Treating childhood acute lymphoblastic leukemia without cranial irradiation. N. Engl. J. Med. 360, 2730-2741.

Pui, C. H., and Evans, W. E. (2006). Treatment of acute lymphoblastic leukemia. N. Engl. J. Med. 354, 166-178.

Pui, C. H., Mullighan, C. G., Evans, W. E., and Relling, M. V. (2012). Pediatric acute lymphoblastic leukemia: where are we going and how do we get there? Blood 120, 1165-1174.

Ramsey, L. B., Bruun, G. H., Yang, W., Trevino, L. R., Vattathil, S., Scheet, P., et al. (2012). Rare versus common variants in pharmacogenetics: SLCO1B1 variation and methotrexate disposition. Genome Res. 22, 1-8.

Relling, M. V., Altman, R. B., Goetz, M. P., and Evans, W. E. (2010). Clinical implementation of pharmacogenomics: overcoming genetic exceptionalism. Lancet Oncol. 11, 507-509.

Relling, M. V., Gardner, E. E., Sandborn, W. J., Schmiegelow, K., Pui, C. H., Yee, S. W., et al. (2011). Clinical Pharmacogenetics Implementation Consortium guidelines for thiopurine methyltransferase genotype and thiopurine dosing. Clin. Pharmacol. Ther. 89, 387-391.

Relling, M. V., and Klein, T. E. (2011). CPIC: Clinical Pharmacogenetics Implementation Consortium of the Pharmacogenomics Research Network. Clin. Pharmacol. Ther. 89, 464-467.

Relling, M. V., Pui, C. H., Cheng, C., and Evans, W. E. (2006). Thiopurine methyltransferase in acute lymphoblastic leukemia. Blood 107, 843-844.

Schrappe, M., Valsecchi, M. G., Bartram, C. R., Schrauder, A., PanzerGrumayer, R., Moricke, A., et al. (2011). Late MRD response determines relapse risk overall and in subsets of childhood T-cell ALL: results of the AIEOP-BFM-ALL 2000 study. Blood 118, 2077-2084.

Schwab, M., and Brauch, H. (2012). Fighting Drug Failure [Online]. Available at: http://www.fightingdrugfailure.net/ [accessed Oct 22, 2012].
Stocco, G., Cheok, M. H., Crews, K. R., Dervieux, T., French, D., Pei, D., et al. (2009). Genetic polymorphism of inosine triphosphate pyrophosphatase is a determinant of mercaptopurine metabolism and toxicity during treatment for acute lymphoblastic leukemia. Clin. Pharmacol. Ther. 85, 164-172.

Stocco, G., Crews, K. R., and Evans, W. E. (2010). Genetic polymorphism of inosinetriphosphate-pyrophosphatase influences mercaptopurine metabolism and toxicity during treatment of acute lymphoblastic leukemia individualized for thiopurine-Smethyl-transferase status. Expert Opin. Drug. Saf. 9, 23-37.

Stocco, G., Yang, W., Crews, K. R., Thierfelder, W. E., Decorti, G., Londero, M., et al. (2012). PACSIN2 polymorphism influences TPMT activity and mercaptopurine-related gastrointestinal toxicity. Hum. Mol. Genet. 21, 4793-4804.

Swen, J. J., Nijenhuis, M., De Boer, A. Grandia, L., Maitland-Van Der Zee, A. H., Mulder, H., et al. (2011). Pharmacogenetics: from bench to byte - an update of guidelines. Clin. Pharmacol. Ther. 89, 662-673.

Swen, J. J., Wilting, I., De Goede, A. L. Grandia, L., Mulder, H., Touw, D. J., et al. (2008). Pharmacogenetics: from bench to byte. Clin. Pharmacol. Ther. 83, 781-787.

Tanaka, Y., Manabe, A., Nakadate, H., Kondoh, K., Nakamura, K., Koh, K., et al. (2012). The activity of the inosine triphosphate pyrophosphatase affects toxicity of 6-mercaptopurine during maintenance therapy for acute lymphoblastic leukemia in Japanese children. Leuk. Res. 36, 560-564.

Thompson, A. J., Fellay, J., Patel, K., Tillmann, H. L., Naggie, S., Ge, D., et al. (2010). Variants in the ITPA gene protect against ribavirin-induced hemolytic anemia and decrease the need for ribavirin dose reduction. Gastroenterology 139, 1181-1189.

Trevino, L. R., Shimasaki, N., Yang, W., Panetta, J. C., Cheng, C., Pei, D., et al. (2009). Germline genetic variation in an organic anion transporter polypeptide associated with methotrexate pharmacokinetics and clinical effects. J. Clin. Oncol. 27, 5972-5978.

von Ahsen, N., Oellerich, M., and Armstrong, V. W. (2008). Characterization of the inosine triphosphatase (ITPA) gene: haplotype structure, haplotype-phenotype correlation and promoter function. Ther. Drug. Monit. 30, 16-22.

Wheeler, H. E., and Dolan, M. E. (2012). Lymphoblastoid cell lines in pharmacogenomic discovery and clinical translation. Pharmacogenomics $13,55-70$.

Yamamoto, K., Okada, Y., Nakamura, K., Hiromura, K., Nojima, Y., and Nakamura, T. (2010). Inosine triphosphate pyrophosphatase 94C $>$ A polymorphism: clinical implications for patients with systemic lupus erythematosus treated with azathioprine. Expert Opin. Drug. Saf. 9, 447-457.

Yang, J. J., Cheng, C., Devidas, M., Cao, X., Campana, D., Yang, W., et al. (2012). Genome-wide association study identifies germline polymorphisms associated with relapse of childhood acute lymphoblastic leukemia. Blood 120, 4197-4204.

Yang, J. J., Cheng, C., Devidas, M., Cao, X., Fan, Y., Campana, D., et al. (2011). Ancestry and pharmacogenomics of relapse in acute lymphoblastic leukemia. Nat. Genet. 43, 237-241.

Yang, J. J., Cheng, C., Yang, W., Pei, D., Cao, X., Fan, Y., et al. (2009). Genome-wide interrogation of germline genetic variation associated with treatment response in childhood acute lymphoblastic leukemia. JAMA 301, 393-403.

Zaza, G., Cheok, M., Krynetskaia, N., Thorn, C., Stocco, G., Hebert, J. M., et al. (2010). Thiopurine pathway. Pharmacogenet. Genomics 20, 573-574.

Conflict of Interest Statement: The authors declare that the research was conducted in the absence of any commercial or financial relationships that could be construed as a potential conflict of interest.

Received: 22 October 2012; accepted: 12 December 2012; published online: 07 January 2013.

Citation: Stocco G, Franca R, Verzegnassi $F$, Londero $M$, Rabusin $M$ and Decorti $G$ (2013) Multilocus genotypes of relevance for drug metabolizing enzymes and therapy with thiopurines in patients with acute lymphoblastic leukemia. Front. Gene. 3:309. doi: 10.3389/fgene.2012.00309

This article was submitted to Frontiers in Pharmacogenetics and Pharmacogenomics, a specialty of Frontiers in Genetics.

Copyright (C) 2013 Stocco, Franca, Verzegnassi, Londero, Rabusin and Decorti. This is an open-access article distributed under the terms of the Creative Commons Attribution License, which permits use, distribution and reproduction in other forums, provided the original authors and source are credited and subject to any copyright notices concerning any third-party graphics etc. 